\title{
Caractérisation de la compétition et de la croissance de Pericopsis elata (Harms) Van Meeuven dans les plantations de la Réserve Forestière de Deng-Deng (Est, Cameroun)
}

\author{
Joseph AMBARA ${ }^{1 *}$, Kadiri Serge BOBO ${ }^{2}$, Juvenal DONFACK DEMESSE ${ }^{2}$ et \\ Antoine David MVONDO-ZE ${ }^{3}$
}
${ }^{1}$ Université de Dschang, Faculté d'Agronomie et des Sciences Agricoles (FASA), Département de foresterie, B.P : 222 Dschang, Cameroun.
${ }^{2}$ Laboratoire de Faune, Aires Protégées, Sylviculture et Technologie du Bois (LAFAPSYTEB), Université de Dschang, B.P : 222 Dschang, Cameroun.
${ }^{3}$ Université de Dschang, Faculté d'Agronomie et des Sciences Agricoles (FASA), Département des Sciences du Sol. BP. 222. Dschang, Cameroun.
*Auteur correspondant ; E-mail: eyengambara@gmail.com; Tel. (00237) 674337005

\author{
Received: $31-10-2020$ \\ Accepted: 26-04-2021 \\ Published: 30-04-2021
}

\section{RESUME}

Pericopsis elata est une espèce ligneuse à grande valeur commerciale menacée d'extinction. Les plantations d'Assamela installées dans la Réserve Forestière de Deng-Deng en 1974 ont été abandonnées après leur mise en place. Dès lors, très peu de connaissances relatives au développement de ces peuplements sont disponibles. L'objectif de la présente étude est de contribuer à la compréhension des conditions de croissance de Pericopsis elata dans la réserve et induire un aménagement efficace. Trois inventaires exhaustifs ont été conduits, au cours desquels les données dendrométriques et structurales ont été collectées. Les indices de compétition de Hegyi, les semi-variogrammes et la distribution spatiale des tiges ont été analysés et interprétés. Les densités actuelles sont sans effet limitant pour la croissance des tiges. Les effets du milieu se révèlent comme étant le facteur qui a plus d'impact sur la croissance des tiges de Pericopsis elata. La plantation de recrû $(33,21 \mathrm{~cm})$, moins sensible à l'effet de la densité en termes de croissance comparée aux plantations des grands layons (26,91 $\mathrm{cm}$ et 26,89 cm), enregistre les meilleures performances de croissance en diamètre. Ainsi, des travaux d'entretien et des éclaircies portées vers une réduction considérable des compétitions interspécifique et intra spécifique sont nécessaires.

(C) 2021 International Formulae Group. All rights reserved.

Mots clés : Indice de Hegyi, méthodes sylvicoles, modes de compétition, plantations équiennes, semivariogramme, Cameroun.

\section{Characterization of the competition and growth of Pericopsis elata (Harms) Van Meeuven in the plantations of the Deng-Deng Forest Reserve (East, Cameroon)}

\begin{abstract}
Pericopsis elata is a valuable woody species threatened with extinction. The Assamela plantations established in the Deng-Deng Forest Reserve in 1974 were abandoned after their establishment. As a result, very little knowledge about the development of these stands is available. The objective of the present study is to
\end{abstract}


contribute to the understanding of the growing conditions of Pericopsis elata in the Reserve and to induce proper management. Three exhaustive inventories were conducted, during which dendrometric data were collected. Hegyi's competition indices, variograms and spatial distribution of stems were developed. Current densities have no limiting effect on stem growth. Environmental effects were found to be the factor that had the greatest impact on the growth of Pericopsis elata stems. The recruiting plantation $(33.21 \mathrm{~cm})$, which is less sensitive to the effect of density in terms of growth compared to the large layon method $(26.91 \mathrm{~cm}$ and $16.89 \mathrm{~cm})$, has the best growth in diameter performance. Thus, maintenance work and thinning leading to a considerable reduction in interspecific and intraspecific competition is necessary.

(C) 2021 International Formulae Group. All rights reserved.

Keywords: Regular plantations, Hegyi's competition index, mode of competition, Silvicultural methods, semivariogram, Cameroon.

\section{INTRODUCTION}

Le Cameroun est couvert pour $42 \%$ de sa surface par des forêts, soit 19916000 d'ha dont 7,3 millions d'ha sont zonées pour l'exploitation durable du bois (FAO, 2011). Cependant, la superficie des forêts camerounaises diminue au rythme d'environ $1000 \mathrm{~km}^{2}$ par an (Kuyper et al., 2001). Face à la diminution de son espace forestier, des programmes de reboisement ont été initiés.

Pericopsis elata (Fabaceae) est une essence noble et à haute valeur commerciale des forêts denses humides semi-sempervirentes africaines en général et du Cameroun en particulier. En raison de sa surexploitation commerciale malheureusement, son aire de distribution est en nette régression (Bourland et al., 2012). Pericopsis elata (Harms) V. Meeuwen (Assamela/Afrormosia) est classée par l'UICN comme une espèce en danger depuis le 11 juin 1992. Elle est inscrite à l'Annexe II de la Convention sur le Commerce International des Espèces de Faune et de Flore Sauvage menacées d'Extinction (CITES) depuis le 03 juin 2019 pour les grumes, le bois de sciage, les placages, les contreplaqués et les bois transformés. Dès lors la gestion de cette espèce revêt un triple enjeu à la fois économique, scientifique et environnemental. A l'instar d'autres grands arbres héliophiles exploités, assurer une régénération suffisante par plantation devrait permettre de pérenniser les populations de Pericopsis elata sur le long terme (Bourland, 2013). Si le commerce international et les utilisations industrielles du bois de Pericopsis elata sont bien documentés, seules des bribes d'information sont connues quant à sa sylviculture (Bourland et al., 2012). En conséquence et eu égard le statut de conservation de cette espèce, la nécessité d'approfondir les recherches dans ce domaine se présente à ce jour comme une urgence.

La maitrise de la sylviculture par plantation d'une espèce n'est autre que la connaissance des interactions qui existent entre les paramètres qui régulent sa croissance. Pour plusieurs auteurs, les faibles densités sont limitantes pour la croissance, alors que les fortes sont saturantes (Le Moguédec et Dhôte, 2012). Dans l'expression de la densité, existe en filigrane la notion d'espacement ou d'écartement lorsqu'il s'agit des peuplements équiens. En effet, dans le cas des peuplements réguliers, les écartements entre les tiges traduisent mieux, sinon exactement, la densité du peuplement. Selon Akouehou et al. (2017), la densité de peuplement réfère à la notion de compétition entre les arbres c'est-à-dire à la demande exercée par deux ou plusieurs organismes pour une ressource commune dans un milieu où ces ressources sont réellement ou potentiellement limitées. Autrement dit, un espacement large des arbres et par ricochet une densité faible engendre une croissance rapide des tiges (Gagné et al., 2012 ; Akouehou et al., 2017) puisque celles-ci bénéficient d'un plus grand accès aux nutriments, à l'eau et à la lumière. Dans le même ordre d'idées, le niveau de compétition entre les arbres augmente avec la densité d'un peuplement (Bérubé-Deschênes et al., 2017). Fonctionnellement, les modes de compétition Pretzsch et Schütze (2016) concernent principalement l'accès aux ressources telles que l'eau, les substances 
nutritives et la lumière; ce qui induit une participation des parties aérienne et souterraine de l'arbre dans ce processus.

La croissance diamétrique des tiges est principalement influencée par les effets de la densité, des interactions entre voisins et en fonction du stade de développement. Plusieurs auteurs s'accordent sur le fait que lorsque la compétition se fait à la fois pour l'eau, les éléments nutritifs et la lumière, les petits arbres ont certains effets adverses sur la croissance des arbres plus grands (Pretzsch et Biber, 2010). La compétition est ainsi bilatérale ou symétrique parce que les systèmes racinaires sont capables d'exploiter les volumes du sol proportionnellement à leur taille, quoique les gros arbres aient un plus grand accès à la fois à la lumière et aux ressources sous-terraines. Par contre, lorsque la compétition est principalement pour la lumière, elle devient fortement unilatérale ou asymétrique en raison de la grande différence de radiation entre la pleine lumière et celle qui passe à travers le feuillage (Pretzsch et Biber, 2010). Autrement dit, en sylviculture de plantation, la compréhension des interactions entre les tiges est un facteur déterminant pour une sylviculture maitrisée d'une espèce.

La présente étude est guidée par l'hypothèse suivant laquelle les densités actuelles de Pericopsis elata dans les plantations de la réserve forestière de DengDeng (RFDD) sont un facteur limitant pour la croissance des tiges. L'objectif est de comprendre et de quantifier les conditions de croissance de Pericopsis elata au passé sans traitements sylvicoles en rapport avec les méthodes sylvicoles, afin de contribuer à la maitrise de la sylviculture de plantation de cette espèce.

\section{MATERIEL ET METHODES \\ Localisation de la Réserve Forestière de Deng-Deng}

La RFDD se situe entre $4^{\circ} 30^{\prime}$ et $5^{\circ} 30^{\prime}$ de latitude Nord, et $13^{\circ} 11^{\circ}$ et $13^{\circ} 30^{\prime}$ de longitude Est. Elle a une superficie de 249000 ha. La RFDD n'est pas située dans l'aire naturelle de Pericopsis elata au Cameroun (Figure 1). Le climat est caractéristique d'un régime équato-guinéen classique à quatre saisons dont deux pluvieuses (une grande saison de pluie de 3,5 mois qui va d'août à minovembre, et une petite saison de pluie de 2,5 mois qui va de mars à mi-mai) et deux sèches (une grande saison sèche de 3,5 mois de minovembre à février, et une petite saison sèche de 2,5 mois de mi-mai à juillet). La moyenne annuelle de la pluviométrie est de $1500 \mathrm{~mm}$ avec des mois écologiquement secs dont la pluviométrie est inférieure à $30 \mathrm{~mm}$ en décembre, janvier et février, avec une température moyenne annuelle variant entre 22 ${ }^{\circ} \mathrm{C}$ et $25^{\circ} \mathrm{C}$.

Les sols de la RFDD sont constitués essentiellement de sols ferralitiques avec trois variantes : les sols ferralitiques typiques (sols ferralitiques rouges dérivés des roches métamorphiques) ; les sols ferralitiques cuirassés (horizons profonds des sols rouges ferralitiques localement cuirassés et mis à nu, concrétions ferrugineuses) nombreux vers le sud; et les sols complexes de savanes (sols remaniés, concrétions et débris de cuirasse) vers le nord (Tchingsabe et al., 2017).

\section{Quelques éléments caractéristiques des plantations d'Assamela de la Réserve Forestière de Deng-Deng}

Les plantations de Pericopsis elata de la RFDD ont été mises en place en 1974 par le Centre Technique Forestier Tropical (CTFT) dans le cadre de son programme de recherche forestière sur l'Assamela. Leurs principales caractéristiques sont présentées dans le Tableau 1.

\section{Collecte des données dendrométriques}

Les données nécessaires pour le présent travail ont été collectées en trois phases, notamment en 2010, 2015 et 2018 dans trois plantations d'Assamela (P741, P745 et P746) installées dans le Bloc-Kébé de la RFDD. Au cours de chacune de ces phases, un inventaire exhaustif pied par pied a été réalisé dans chaque plantation. Les travaux d'inventaire étaient ponctués par quatre principales articulations dont le nettoyage, le marquage, le comptage et la collecte des données dendrométriques. 
Le nettoyage, consistait à ouvrir les lignes de plantation pour faciliter la progression entre les lignes et les colonnes. Le marquage consistait à identifier toutes les tiges de Pericopsis elata existantes et à les marquer à la peinture blanche sur la face Est. L'opération de comptage consistait à attribuer un numéro d'identification à chaque tige. Dans cette opération de comptage, les tiges mortes étaient prises en compte. A l'issue de l'inventaire, le Diamètre à hauteur de poitrine (Dhp) et la hauteur totale de chaque tige ont été relevés à l'aide du ruban dendrométrique et du Relascope de Bitterlich.

\section{Distribution spatiale des tiges}

La méthode des quadrats a été utilisée pour décrire la distribution des tiges dans chaque plantation. Ainsi, les plantations P741, P745 et P746 ont été subdivisées en quadrats de $200 \mathrm{~m}^{2}, 360 \mathrm{~m}^{2}$ et $480 \mathrm{~m}^{2}$ respectivement. Le principe étant de tester la forme de la distribution des tiges dans la plantation sous l'hypothèse nulle que la distribution n'est pas significativement différente d'une distribution aléatoire. La forme de la distribution spatiale des tiges est déterminée par l'Indice de Concentration (IC) obtenu par la formule suivante.

Indice de Concentration $=\frac{V(D)}{D}$

Avec $\mathrm{D}=$ la densité moyenne dans chaque quadrat; $\mathrm{V}(\mathrm{D})$ = la variance du nombre de tiges par quadrat.

IC $\approx 1$ : distribution est aléatoire ; IC $>1$ : distribution est concentrée et IC $<1$ : Distribution régulière.

\section{Analyse variographique}

L'analyse variographique a été utilisée dans le but d'explorer l'autocorrélation des tiges dans les plantations. Concrètement, l'idée fondamentale est que deux tiges rapprochées ou situées l'une près de l'autre dans une plantation devraient posséder des caractéristiques similaires que celles éloignées. L'outil convoqué pour cela est le semivariogramme qui décrit l'évolution de la semi- variance en fonction de la distance entre les mesures (Dhp et hauteur) et permet ainsi d'étudier le lien spatial entre les données (Kouassi et al., 2012). A partir des données dendrométriques (Dhp et hauteur), le semivariogramme $\gamma(\mathrm{h})$ a été estimé de la manière suivante :

$\gamma(h)=\frac{1}{2} \frac{\sum_{1}^{N(h)}[Z(x)-Z(x+h)]^{2}}{N(h)}$

Avec: $\mathrm{Z}(\mathrm{x})$ est la valeur du Dhp ou de la hauteur au point $\mathrm{x}$ de la plantation; $\mathrm{h}=$ une distance entre deux tiges $; N(h)=$ le nombre de paires dont les tiges sont espacées de $h$.

L'analyse des propriétés des semivariogrammes a permis d'estimer et de qualifier les types de corrélation existant entre les tiges dans chaque plantation. Les interactions entre les tiges ont été abordées en considérant que celles-ci s'expriment dans chaque plantation sous la forme d'une structure aléatoire. Afin d'apprécier simultanément les interactions et la dynamique des plantations, les semi-variogrammes expérimentaux ont été présentés suivant quatre principales directions, notamment $0 \circ, 45^{\circ}$, $90^{\circ}$ et $135^{\circ}$. Contrairement aux plantations P745 et P746, les semi-variogrammes expérimentaux et théoriques ont été construits pour la plantation P741. En effet, compte tenu de la méthode sylvicole des plantations P745 et P746 (Méthode des grands layons), les interactions intra spécifiques entre les colonnes sont inexistantes ou non pertinentes. Fort de cela, les semi-variogrammes expérimentaux ont été construits individuellement pour chaque ligne de ces plantations.

L'analyse de l'allure globale des points d'une part et la valeur des premiers de chaque semi-variogramme expérimental d'autre part ont permis d'identifier et de caractériser les types d'interactions (coopération, compétition, effet de densité) existants entre les tiges de chaque plantation. D'après Bachacou et Lemoine (1976), s'il y'a coopération, le variogramme croîtra jusqu'à un palier (Figure 2a), sachant qu'au-delà d'une certaine distance qu'indique la «portée », l'effet de coopération 
n'est plus sensible d'une tige à l'autre. L'effet du milieu se traduit par un semi-variogramme de même type, à la différence que la portée ici indique la dimension moyenne de zones d'égale fertilité dans la direction où le semivariogramme a été calculé. Lorsqu'il y a concurrence (Figure $2 \mathrm{~b}$ et $2 \mathrm{c}$ ), les voisins immédiats d'une tige auront en moyenne une croissance réduite si celle-ci est vigoureuse, et une croissance forte dans le cas contraire. Le premier point du semi-variogramme est donc plus élevé que le deuxième et les suivants, produisant un pic caractéristique. Dans le même ordre d'idée, l'interprétation de la valeur de la portée lors des ajustements des semivariogrammes expérimentaux a permis de quantifier les interactions entre les tiges dans la plantation de recrû.

\section{Surface terrière et diamètre quadratique moyen}

La surface terrière $(G)$ est la somme de la section transversale à $1,30 \mathrm{~m}$ au-dessus du sol de toutes les tiges. Elle est calculée par la formule suivante :

$G=\frac{\pi}{4 \times S \times 10^{4}} \sum_{i=1}^{n} D h p_{i}^{2}$

$\mathrm{S}=$ superficie de la plantation $\left(\mathrm{m}^{2}\right) ; \mathrm{Dhp}_{\mathrm{i}}=$ diamètre à $1,30 \mathrm{~m}$ de la tige $\mathrm{i}(\mathrm{m}) ; G=$ s'exprime en $\mathrm{m}^{2} / \mathrm{ha}$.

Le diamètre quadratique moyen $\left(D_{q}\right)$, qui est le Dhp de l'arbre de surface terrière moyenne de chaque plantation, a été estimé par la formule mathématique suivante :

$D_{q}=200 \sqrt{G / \pi \times N}$

$\mathrm{N}=$ Densité de la plantation en (t/ha).

\section{Indice de compétition de Hegyi}

La compétition a été appréciée dans chaque plantation à travers l'indice de Hegyi (1974). Cet indice dépendant de la distance, est basé sur les ratios de taille pondérés par la distance. Partant du postulat suivant lequel le diamètre est proportionnel à la hauteur d'une tige, la concurrence (compétition) a été appréciée suivant le statut social de l'arbre sujet et en fonction du statut des compétiteurs (Dominant, Co-dominant et Dominé). L'indice de Hegyi se calcule de la manière suivante :

$I C_{i}=\sum_{j=1}^{n} \frac{D h p_{j}}{D_{h p_{i}} * \operatorname{Dist}_{i j}}$

$I C_{i}=$ Indice de Compétition de Hegyi de la tige $\mathrm{i} ; D h p_{i}$ et $D h p_{j}$ : diamètre à $1,30 \mathrm{~m}$ des tiges i et $\mathrm{j}$ respectivement $; D_{i s t_{i j}}=$ Distance entre les tiges $i$ et $j$.

Les indices de compétition de Hegyi ont été estimés pour chaque plantation suivant que la compétition est exercée par un arbre dominant, un co-dominant ou alors par un arbre dominé.

\section{Analyses statistiques}

Le test de Khi-deux a été utilisé pour conclure au caractère aléatoire ou non des distributions des tiges. La modélisation et la construction des semi-variogrammes théoriques ont été réalisées à l'aide du logiciel Rstudio (R Core Team, 2020). Les tests de comparaison des moyennes des échantillons indépendants ont été utilisés pour juger la significativité des différences observées entre les trois plantations au travers des moyennes calculées pour chacune de leur caractéristique. Dans le même ordre d'idées, le test d'adéquation de Khi-deux a été utilisé pour modéliser la distribution des tiges dans chaque plantation, et l'analyse des variances à un facteur (ANOVA) a été utilisée pour comparer l'intensité des corrélations entre les compétiteurs et leur statut social dans les différentes plantations. Lorsque les conditions d'application de ANOVA n'étaient pas vérifiables (normalité et homogénéité des variances), le test non paramétrique de Kruskal et Wallis était utilisé pour tester la comparaison des différences des diamètres moyens entre les lignes de plantation des P745 et P746. L'étude des relations entre les densités, les surfaces terrières et les diamètres quadratiques a été effectuée à travers le coefficient de corrélation (r) estimé avec le logiciel Rstudio (R Core Team, 2020). 
J. AMBARA et al. / Int. J. Biol. Chem. Sci. 15(2): 559-577, 2021

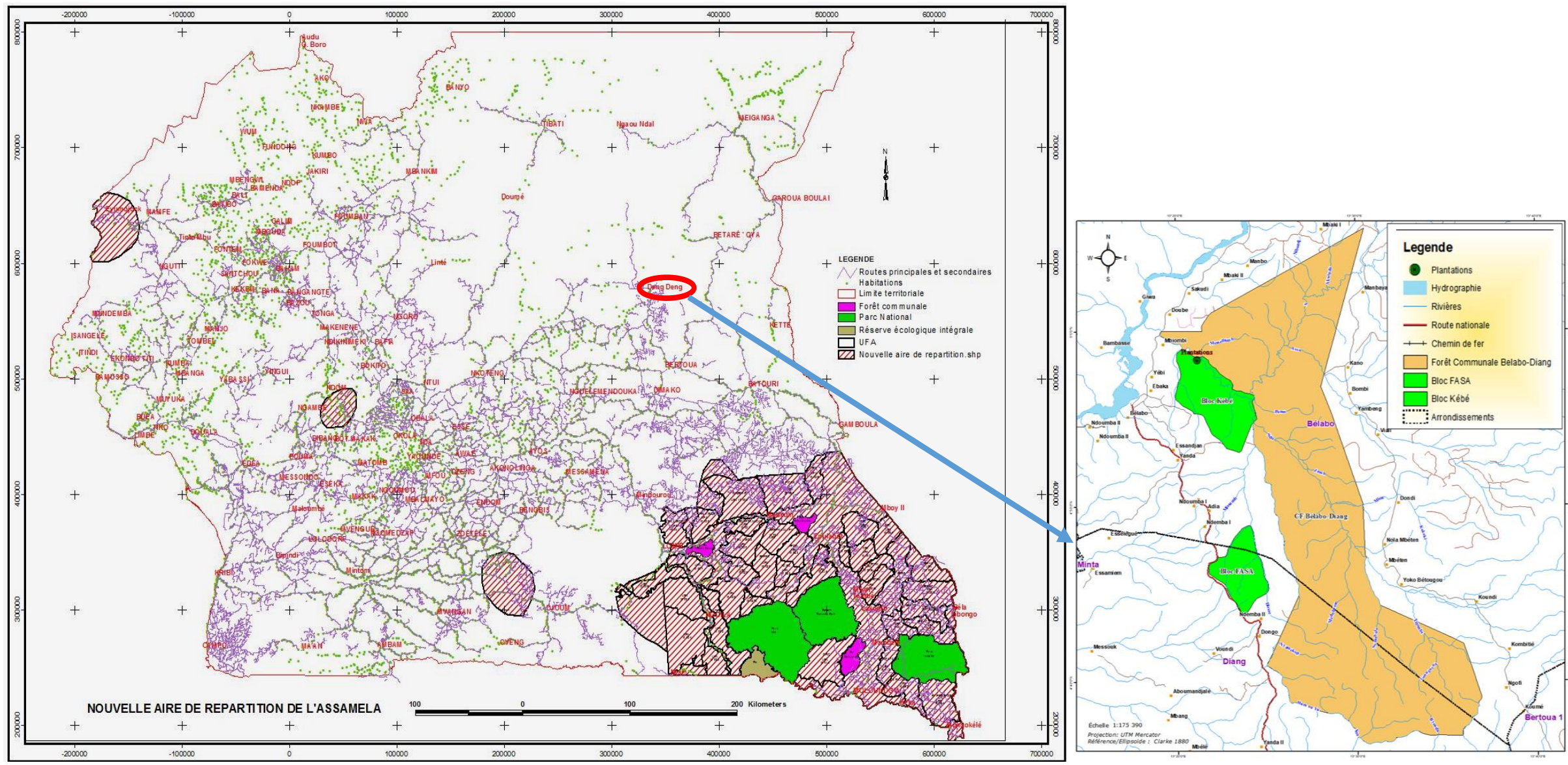

Figure 1 : Localisation de la Réserve Forestière de Deng-Deng et des plantations de Pericopsis elata. 
Tableau 1 : Caractéristiques des plantations d'Assamela de la Réserve Forestière de Deng-Deng.

\begin{tabular}{lccccc}
\hline Plantation & $\begin{array}{c}\text { Superficie } \\
\text { (ha) }\end{array}$ & Ecartement & $\begin{array}{c}\text { Effectif } \\
\text { initial }\end{array}$ & $\begin{array}{c}\text { Méthode } \\
\text { Sylvicole }\end{array}$ & Type de plants \\
\hline P741 & 1 & $5 \mathrm{~m} \times 4 \mathrm{~m}$ & 500 & Recrû & Stumps courts + Plants en sachets \\
\hline P745 & 2 & $15 \mathrm{~m} \times 3 \mathrm{~m}$ & 512 & $\begin{array}{c}\text { Grands } \\
\text { layons }\end{array}$ & Plants en sachets \\
\hline P746 & 2 & $20 \mathrm{~m} \times 3 \mathrm{~m}$ & 384 & $\begin{array}{c}\text { Grands } \\
\text { layons }\end{array}$ & Stumps courts \\
\hline
\end{tabular}

Source : Grison (1979).
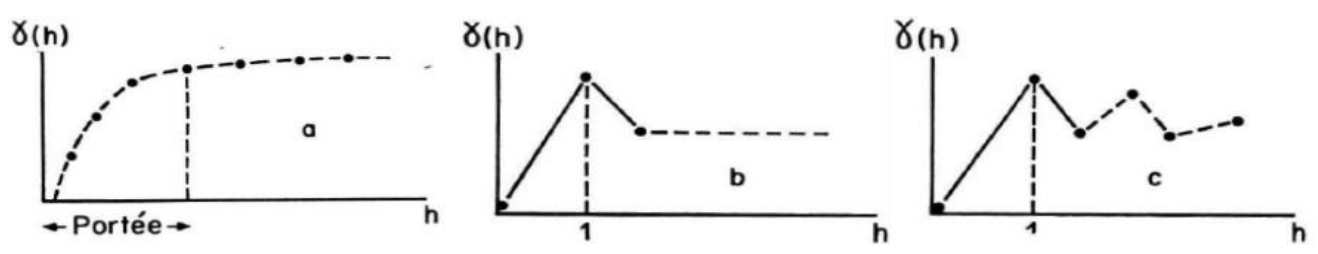

Figure 2 : Formes de semi-variogramme suivant le type d'interaction : coopération (a) et compétition (b et c).

\section{RESULTATS}

\section{Paramètres structuraux et dendrométriques des plantations}

Les paramètres structuraux et dendrométriques estimés à partir des mesures effectuées dans les plantations sont présentés dans le Tableau 2. Ce Tableau montre que l'espace vital ou l'espace de croissance de chaque tige des plantations P745 $\left(45 \mathrm{~m}^{2}\right)$ et P746 $\left(60 \mathrm{~m}^{2}\right)$ est respectivement le double et le triple de celui de la plantation P741 $\left(20 \mathrm{~m}^{2}\right)$. Par ailleurs, le Tableau 2 présente clairement le contraste qui existe dans les tendances entre les densités, les diamètres quadratiques et les surfaces terrières dans les plantations de grand layon.

En effet, les tests de corrélation (Tableau 3) indique qu'il existe une corrélation négative forte entre les densités et les surfaces terrières d'une part et entre les densités et les diamètres quadratiques de chaque plantation. Autrement dit, la baisse des effectifs au fil des années dans chacune des plantations induit une augmentation considérable de la surface terrière. Ainsi, pendant que la mortalité naturelle réduit les effectifs de la P741 de 260 à 258 puis à 243 , on observe une augmentation de la surface terrière de $17,88 \mathrm{~m}^{2} / \mathrm{ha}, 20,23$ $\mathrm{m}^{2} /$ ha et $21,04 \mathrm{~m}^{2} / \mathrm{ha}$.
Dans le même ordre d'idées, on constate à l'échelle de chaque plantation que malgré la diminution de la densité, on dénote une augmentation en diamètre. Ainsi, les densités de la P745 sont passées de 142 tiges/ha à 136 tiges/ha puis 132 tiges/ha, pour une augmentation du diamètre quadratique moyen évaluée à $23,85 \mathrm{~cm}, 25,87 \mathrm{~cm}$ et $26,11 \mathrm{~cm}$ respectivement. En termes de proportion, une diminution de 6 tiges et 4 tiges entraine ainsi une augmentation substantielle du diamètre quadratique évaluée à $2,02 \mathrm{~cm}$ et $1,04 \mathrm{~cm}$ respectivement. Les mêmes tendances sont observées dans la plantation P746 avec une diminution de la densité estimée à 131, 128 puis 122 tiges/ha qui a induit une augmentation du diamètre quadratique estimée à $0,53 \mathrm{~cm}$ et $0,44 \mathrm{~cm}$ respectivement.

Toutefois, lorsqu'on considère individuellement chaque plantation et indépendamment de l'âge, le test de corrélation indique une corrélation positive forte $(r=1)$ entre les densités des trois plantations et leurs croissances en diamètre. En effet, on constate que la plantation P741 qui a les densités les plus élevées quel que soit l'âge comparée aux plantations P745 et P746 enregistre également les croissances en diamètre les plus élevées. 


\section{Modélisation de la distribution des tiges dans les plantations}

Les résultats des tests de la forme des distributions des tiges de chaque plantation sont présentés dans le Tableau 4. Ce tableau indique que les valeurs des indices de concentration de chaque plantation sont inférieures à 1. Toutefois, l'hypothèse nulle $\left(\mathrm{H}_{0}\right)$ est acceptée pour les plantations P745 $\left(\chi_{\text {cal }}=1,73<\chi_{\text {tab }}=9,49\right)$ et P746 $\left(\chi_{\text {cal }}=4,35\right.$ $\left.<\chi_{\text {tab }}=9,49\right)$ au seuil critique de $5 \%$. Ainsi, la forme de distribution des tiges des plantations P745 et P746 est aléatoire (Figure 3). Par contre, comme illustré dans la Figure 4, la forme de la distribution de la plantation P741 est régulière $\left(\chi_{c a l}=16,67>\chi_{\text {tab }}=11,07\right)$.

Nonobstant le caractère héliophile de Pericopsis elata, on observe que la distribution des arbres à l'intérieur des plantations P745 et P746 ne présente pas véritablement un effet de bordure (Figue 3). Par ailleurs, la Figure 3 illustre une croissance en diamètre très variable d'une ligne de plantation à une autre. Ce qui renforce le caractère aléatoire des formes de distribution des tiges de ces deux plantations. Le test de Kruskal-Wallis réalisé au seuil de $5 \%$ indique qu'il n'y a pas de différence significative entre les diamètres moyens des lignes $\left(\chi_{0,05}^{2}=11,07>6,48^{\mathrm{E}}-8\right.$ pour la $\mathrm{P} 746$ et $\chi_{0,05}^{2}=14,07>0,0013$ pour la P745).

La distribution des tiges dans la plantation P741 telle que l'illustre la Figure 4 n'indique pas clairement un effet de bordure. Par ailleurs, la Figure 4 illustre un phénomène de mortalité successive, c'est-à-dire la mortalité de plusieurs tiges sur une ligne de manière successive. Cela est bien visible sur la première et la $9^{\text {ième }}$ ligne de plantation qui enregistrent des taux de mortalité de $90 \%$ et $75 \%$, soit 9 tiges et 8 tiges sur 10 respectivement. Dans le même ordre d'idées, des tiges dont le diamètre est inférieur ou égal à $20 \mathrm{~cm}$, soit les dominés, se retrouvent sans voisins immédiats sur toutes les directions.

\section{Variogrammes expérimentaux et théoriques des plantations}

La Figure 5 illustre les sémivariogrammes expérimentaux et théoriques de la plantation P741, et les sémi-variogrammes expérimentaux de chaque ligne de plantation pour les plantations P745 et P746 sont illustrés par la Figure 6.

Les formes des semi-variogrammes expérimentaux de la plantation P741 sont similaires suivant les quatre directions choisies $\left(0^{\circ}, 45^{\circ}, 90^{\circ}\right.$ et $\left.135^{\circ}\right)$ et cela indépendamment de la variable d'intérêt (Dhp ou Hauteur). À contrario, on constate une forte variabilité des semi-variogrammes des lignes des plantations P745 et P746. Par ailleurs, les premiers points des semi-variogrammes expérimentaux de toutes les plantations indépendamment de la direction ne sont pas les plus élevés. On note également une variabilité de la variance entre les lignes de plantation des plantations P745 et P746 (Figure 6). Les sémi-variogrammes théoriques ajustés au modèle sphérique (Figure 5) de la plantation P741 indique une variation de la portée en fonction de la variable, soit 3,9 $\mathrm{m}$ pour le Dhp et $4,6 \mathrm{~m}$ pour la hauteur.

\section{Indice de compétition par plantation en fonction du statut social de l'arbre sujet}

La Figure 7 illustre la variation des indices de compétition de Hegyi en fonction de la distance entre le compétiteur et l'arbre sujet (dominant, co-dominant ou dominé) dans chaque plantation. Globalement et à l'observation, l'écart (différence des valeurs de l'indice de Hegyi) entre les compétiteurs dominants et co-dominants d'une part, et entre les compétiteurs dominants et dominés d'autre part, est plus important dans la plantation P741 comparé aux plantations P745 et P746 (Figure 7). Dans le même ordre d'idées, on note que l'écart entre les dominants, les co dominants et les dominés est régulier dans les plantations P745 et P746. Par ailleurs, l'analyse de variance au seuil de signification de 5\% indique qu'il n'y a pas de différence significative entre les compétiteurs dans chaque plantation $(p=0,20>0,05 ; p=0,52>$ 0,$05 ; p=0,71>0,05$ respectivement pour les plantations P741, P745 et P746). En outre, les indices de compétition de Hegyi dans les trois plantations sont tous inférieurs à 1 . Autrement dit, la compétition entre les tiges de Pericopsis elata au stade actuel de croissance de chacune des plantations est non significative. 
J. AMBARA et al. / Int. J. Biol. Chem. Sci. 15(2): 559-577, 2021

Tableau 2: Paramètres dendrométriques et structuraux des plantations P741, P745 et P746.

\begin{tabular}{|c|c|c|c|c|c|c|c|c|c|c|}
\hline Plantation & $\begin{array}{c}\text { Effectif } \\
\text { initial }\end{array}$ & $\begin{array}{c}\text { Espace } \\
\text { vital }\left(\mathbf{m}^{2}\right)\end{array}$ & Année & $\begin{array}{c}\text { Nombre de } \\
\text { tiges }\end{array}$ & $\mathbf{N}($ tiges/ha) & Dmoy (cm) & $\mathrm{D}_{\mathrm{q}}(\mathbf{c m})$ & $\begin{array}{c}\mathbf{G} \\
\left(\mathbf{m}^{2} / \mathbf{h a}\right)\end{array}$ & $\begin{array}{c}\text { Taux de } \\
\text { survie }(\%)\end{array}$ & $\begin{array}{c}\text { AAM } \\
(\mathbf{c m} / \mathbf{a n})\end{array}$ \\
\hline \multirow{3}{*}{ P741 } & \multirow{3}{*}{500} & \multirow{3}{*}{20} & 2010 & 260 & 260 & $25,87 \pm 14,38$ & 29,6 & 17,88 & 52 & 0,72 \\
\hline & & & 2015 & 258 & 258 & $27,88 \pm 14,90$ & 30,61 & 20,23 & 51,6 & 0,68 \\
\hline & & & 2018 & 243 & 243 & $29,59 \pm 15,09$ & 33,21 & 21,04 & 48,6 & 0,67 \\
\hline \multirow{3}{*}{ P745 } & \multirow{3}{*}{512} & \multirow{3}{*}{45} & 2010 & 284 & 142 & $20,61 \pm 12,01$ & 23,85 & 3,17 & 53 & 0,57 \\
\hline & & & 2015 & 271 & 136 & $22,84 \pm 12,15$ & 25,87 & 3,56 & 52,9 & 0,56 \\
\hline & & & 2018 & 264 & 132 & $23,97 \pm 12,24$ & 26,91 & 3,75 & 51,6 & 0,54 \\
\hline \multirow{3}{*}{ P746 } & \multirow{3}{*}{384} & \multirow{3}{*}{60} & 2010 & 261 & 131 & $20,76 \pm 9,79$ & 22,95 & 2,7 & 67,9 & 0,56 \\
\hline & & & 2015 & 255 & 128 & $23,5 \pm 10,12$ & 25,58 & 3,275 & 66,4 & 0,57 \\
\hline & & & 2018 & 243 & 122 & $24,83 \pm 10,32$ & 26,89 & 3,45 & 63,3 & 0,56 \\
\hline
\end{tabular}

Dmoy = Diamètre moyen, le chiffre après « \pm » représente l'écart-type $; \mathbf{N}=$ Densité; $\mathbf{D}_{\mathbf{q}}=$ Diamètre quadratique moyen ; $\mathbf{G}=$ Surface terrière $; \mathbf{A A M}=$ Accroissement Annuel Moyen $;$ Espace vital/disponible $=$ Espace initial disponible pour chaque arbre pour sa croissance ; il est fonction de l'écartement initial entre les tiges. 
Tableau 3 : Valeurs des coefficients de corrélation entre la densité et le diamètre quadratique et entre la densité et la surface terrière pour les trois plantations P741, P745 et P746.

\begin{tabular}{cccc}
\hline \multirow{2}{*}{ P741 } & & Diamètre quadratique $\left(\mathbf{D}_{\mathbf{q}}\right)$ & Surface terrière $(\mathbf{G})$ \\
\cline { 2 - 4 } & Densité & $\mathrm{r}=-0,99$ & $\mathrm{r}=-0,77$ \\
& & $\mathrm{p}=0,04$ & $\mathrm{p}=0,03$ \\
\hline \multirow{2}{*}{ P745 } & & Diamètre quadratique $\left(\mathbf{D}_{\mathbf{q}}\right)$ & Surface terrière $(\mathbf{G})$ \\
\cline { 2 - 4 } & Densité & $\mathrm{r}=-1$ & $\mathrm{r}=-1$ \\
& & $\mathrm{p}=0,09$ & $\mathrm{p}=0,003$ \\
\hline \multirow{2}{*}{ P746 } & & Diamètre quadratique $\left(\mathbf{D}_{\mathbf{q}}\right)$ & Surface terrière $(\mathbf{G})$ \\
\cline { 2 - 4 } & Densité & $\mathrm{r}=-0,88$ & $\mathrm{r}=-0,93$ \\
& & $\mathrm{p}=0,16$ & $\mathrm{p}=0,007$ \\
\hline
\end{tabular}

Tableau 4 : Paramètres des tests de la forme de distribution des tiges des plantations.

\begin{tabular}{lccccccc}
\hline Plantations & Nombre de quadrats & $\mathbf{D}$ & $\mathbf{V}(\mathbf{D})$ & IC & $\chi_{\text {cal }}$ & DDL & $\chi_{\mathbf{0}, \mathbf{0 5}}^{\mathbf{2}}$ \\
\hline P741 & 50 & 4,96 & 3,79 & 0,76 & 16,67 & 5 & 11,07 \\
\hline P745 & 64 & 4,06 & 3,01 & 0,74 & 1,73 & 4 & 9,49 \\
\hline P746 & 48 & 5,13 & 2,54 & 0,50 & 4,35 & 4 & 9,49 \\
\hline
\end{tabular}

$\mathbf{D}$ = Densité moyenne; $\mathbf{V}(\mathbf{D})$ = la variance du nombre de tiges par quadrat : $\mathbf{I C}=$ Indice de Concentration.

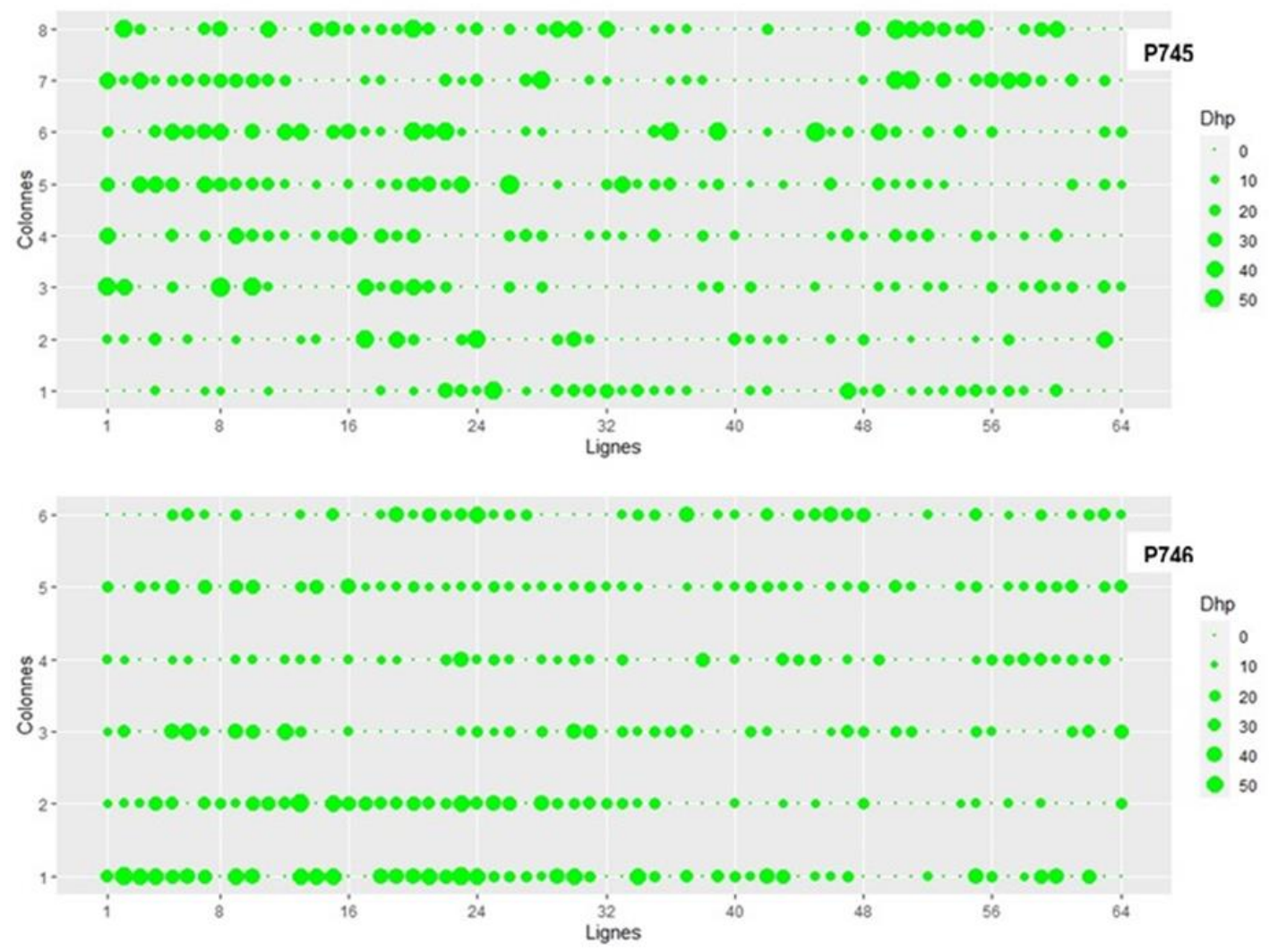

Figure 3 : Distribution spatiale aléatoire des tiges dans les plantations P745 et P746. 


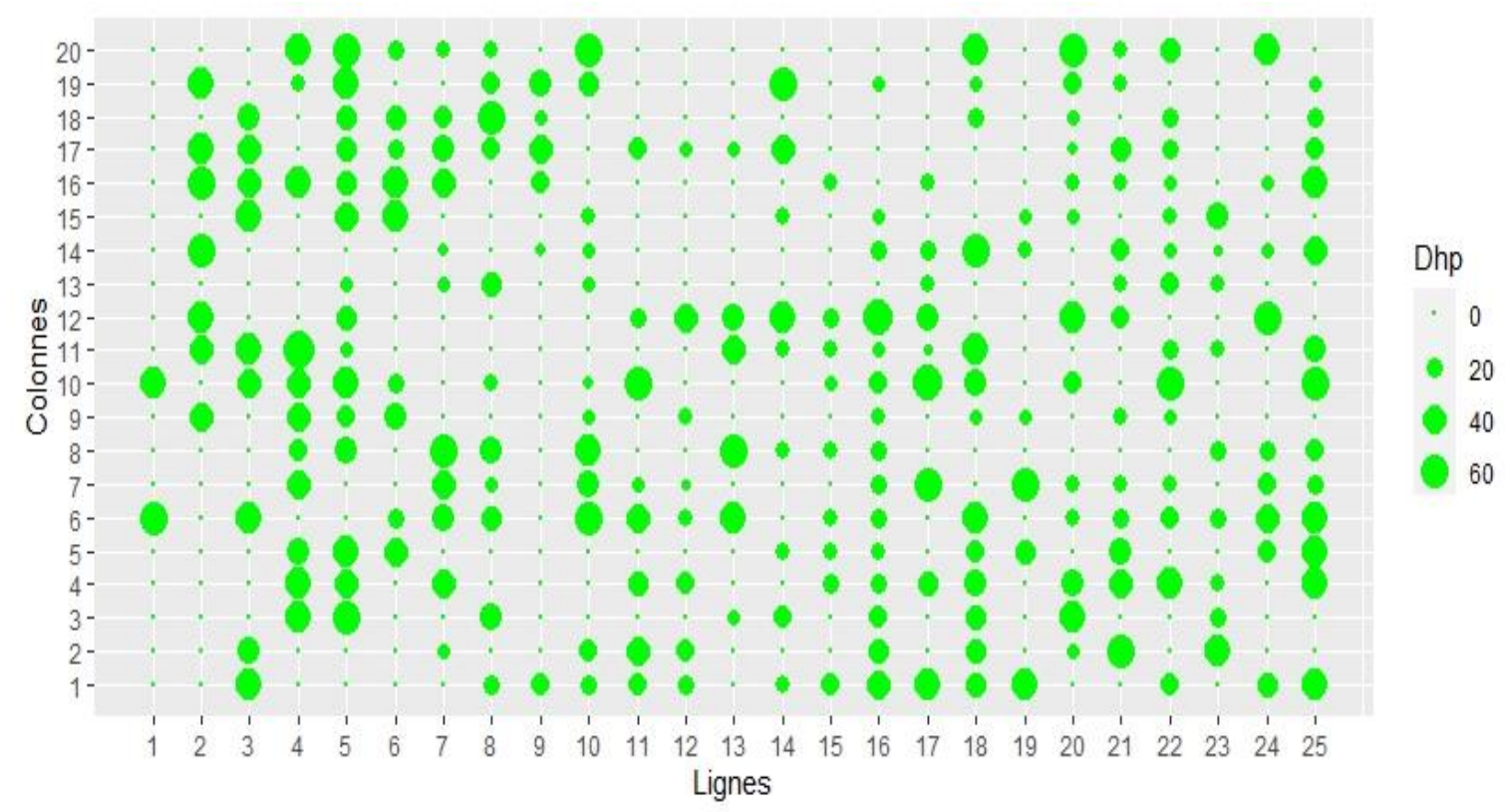

Figure 4 : Distribution régulière des tiges dans la plantation P741.

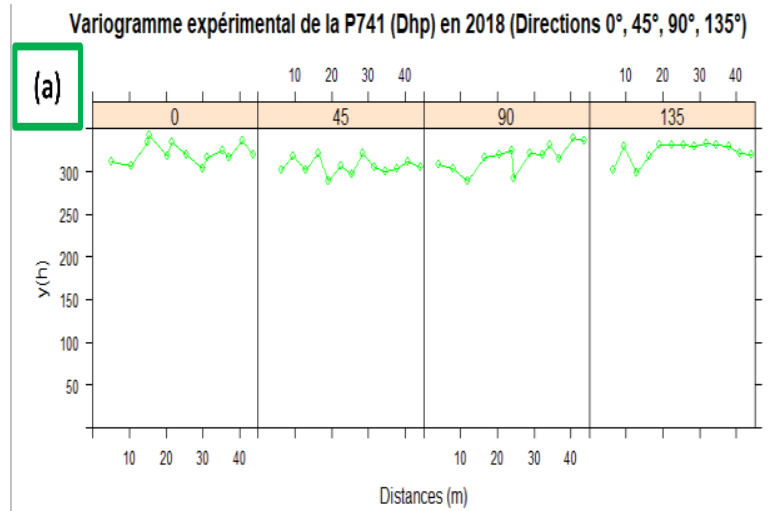

Variogrammes de la P741 (2018) ajustés avec le modèle sphérique
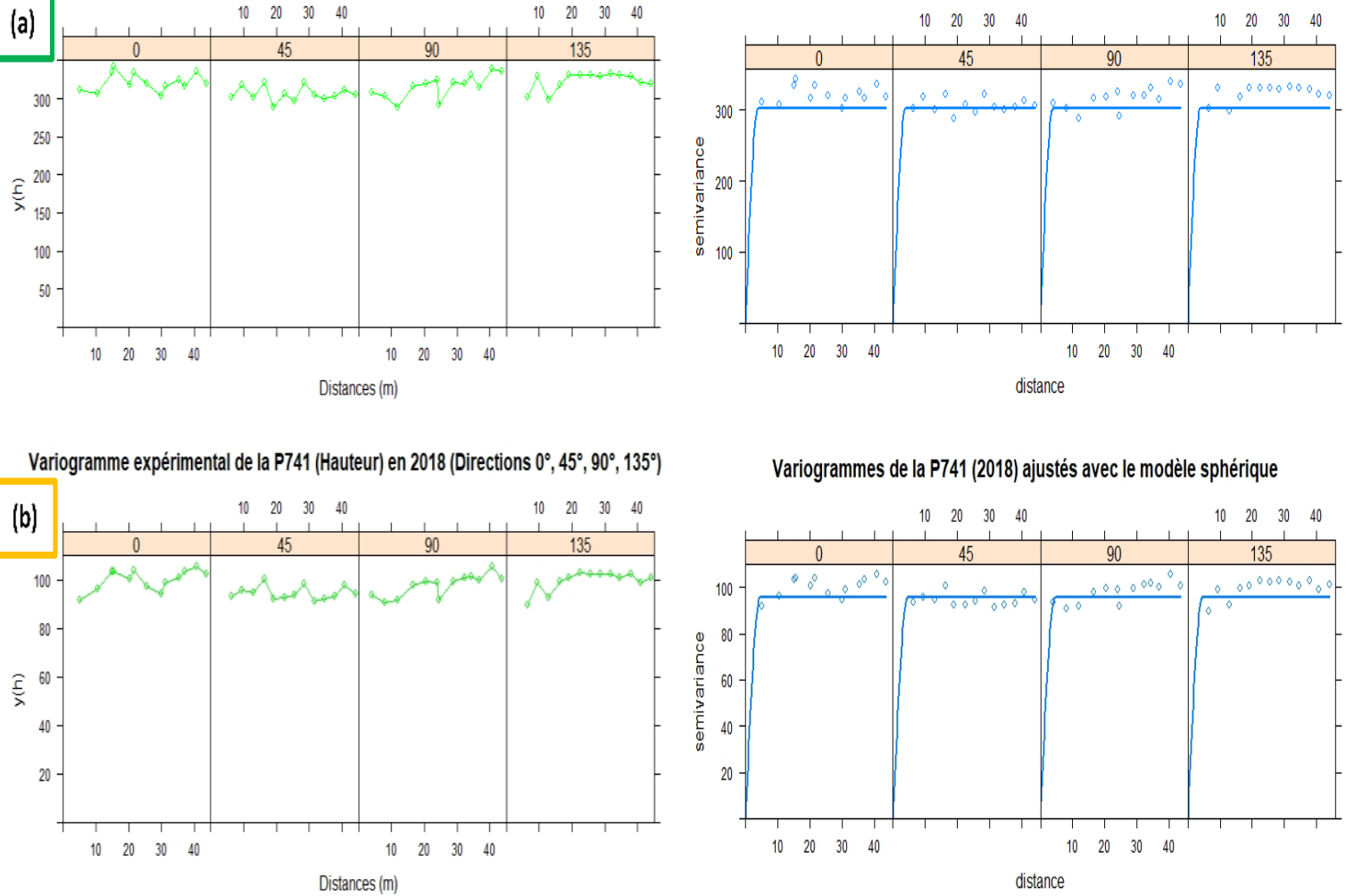

Variogrammes de la P741 (2018) ajustés avec le modèle sphérique

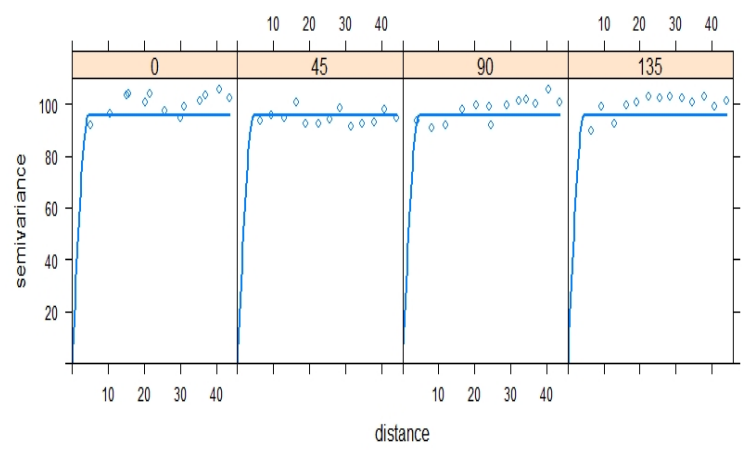

Figure 5 : Variogrammes expérimentaux (gauche) et théoriques (à droite) de la P741 en fonction du Dhp (a) et de la hauteur (b) suivant les directions $\left(0^{\circ}, 45^{\circ}, 90^{\circ}\right.$ et $\left.135^{\circ}\right)$. 
(a) P745 (Ligne 1

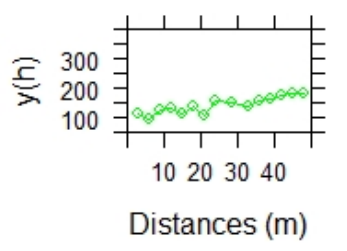

(e) P745 (Ligne 5)

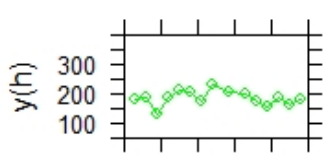

10203040

Distances (m) (b) P745 (ligne 2)

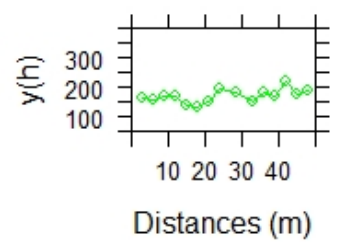

(f) P745 (Ligne 6)

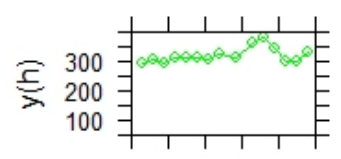

10203040

Distances (m) (c) P745 (Ligne 3)

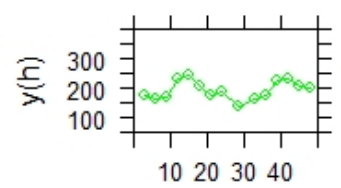

Distances (m) (d) P745 (Ligne 4)

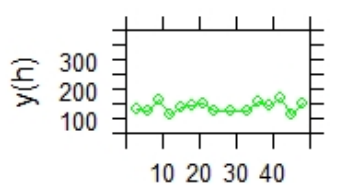

Distances $(\mathrm{m})$ (g) P745 (Ligne 7)

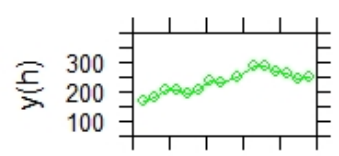

10203040

Distances $(m)$ (h) P745 (Ligne 8)

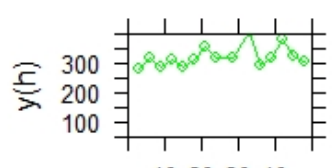

10203040

Distances (m) (a) P746 (Ligne 1)

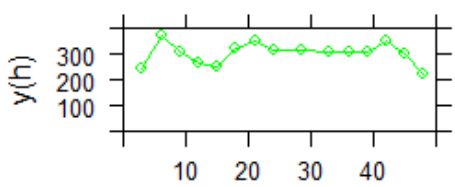

Distances (m)

(d) P746 (Ligne 4)

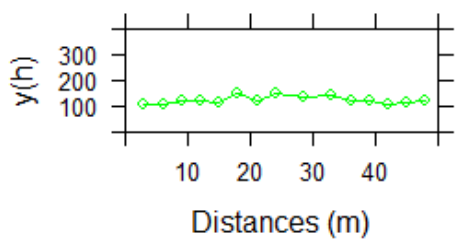

(b) P746 (Ligne 2)

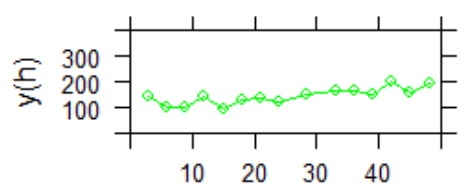

Distances $(m)$

(e) P746 (Ligne 5)

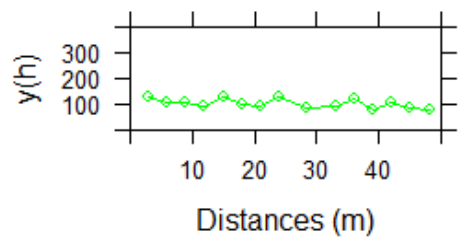

(c) P746 (Ligne 3)

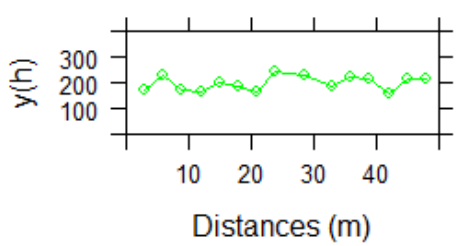

(f) P746 (Ligne 6)

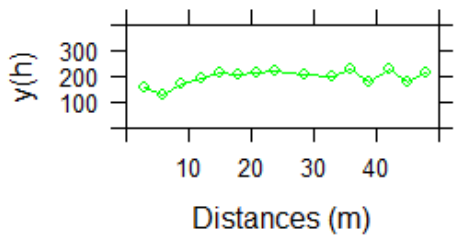

Figure 6 : Variogrammes expérimentaux des lignes des plantations P745 (lignes 1 à 8) et P746 (lignes 1 à 6). 

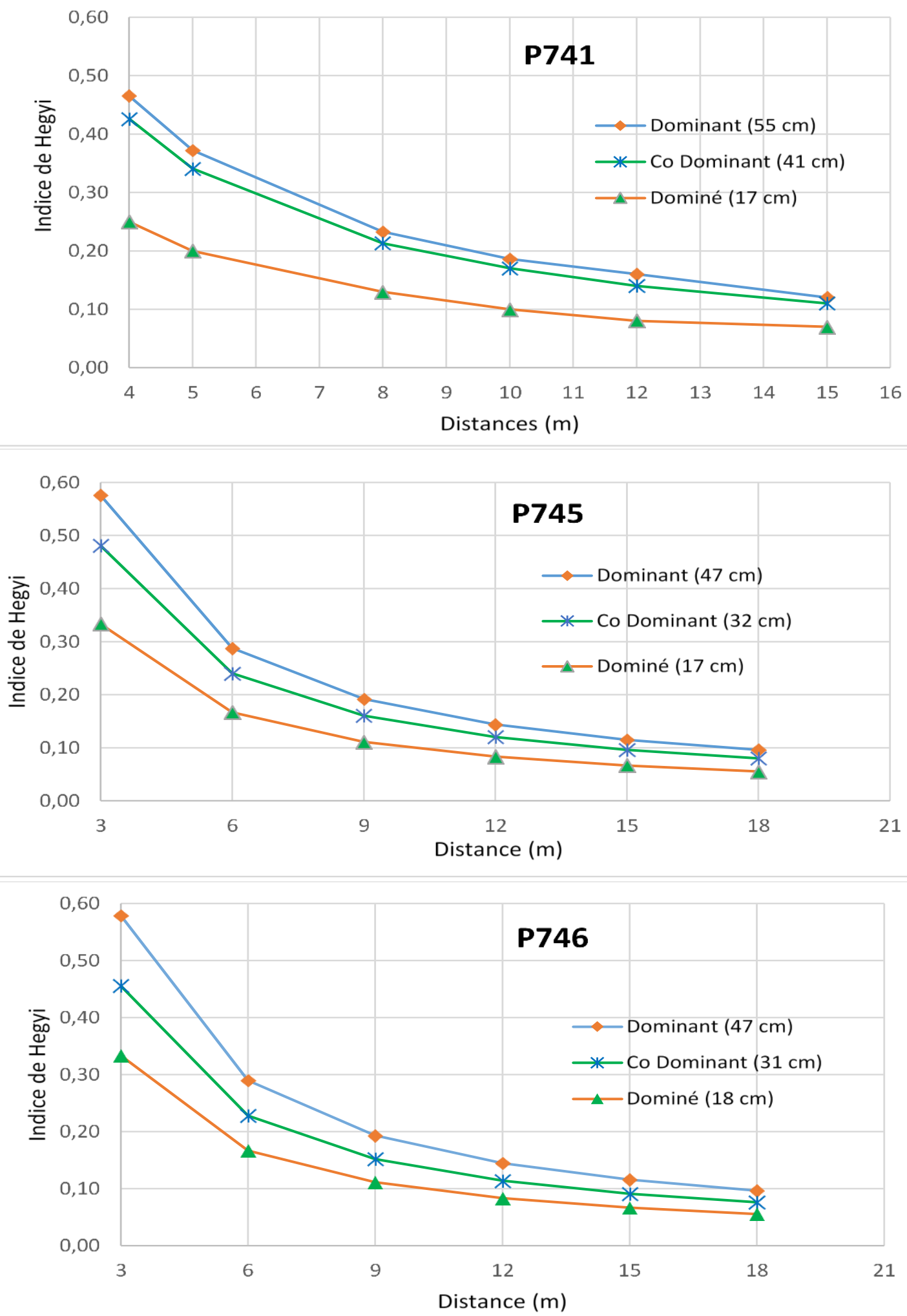

Figure 7 : Courbes des indices de compétition de Hegyi en fonction du statut social des arbres dans les plantations P741, P745 et P746. 


\section{DISCUSSION}

\section{Effet de la densité des plantations sur la croissance}

La densité des peuplements joue un rôle important dans la croissance des arbres en conditionnant le partage des ressources disponibles entre les différents arbres du peuplement et donc le niveau de concurrence individuelle (Périé et al., 2006). Les résultats du présent travail montrent que la croissance en diamètre dans la plantation P741 est plus importante comparée à celles des plantations de grands layons (P745 et P746) qui ont par ailleurs des densités moins importantes que celle de la plantation P741. Ce résultat est en parfaite accord avec les travaux de Ngueguim et al. (2012) qui ont établit également que les plantations de recrû ayant des densités les plus élevées enregistraient les croissances les plus fortes. La concordance des résultats qui se dégage à ce niveau confirme par ailleurs la relation entre les méthodes sylvicoles et la croissance des tiges. Il s'agit de la mise en évidence de l'effet de la densité initiale et par ricochet de l'espacement initial sur la croissance des tiges. En effet, les résultats obtenus dans la présente étude indiquent que l'espacement unitaire des tiges ou l'espace vital disponible dans les différentes plantations par ordre croissant va de la plantation P741 à la plantation P746 (le plus élevé) en passant par la P745 (intermédiaire). Ce résultat rejoint les travaux Medhurst et al. (2001), qui ont conclu que l'accroissement cumulatif en surface terrière diminuait significativement après une diminution de la densitédans les peuplements Eucalyptus nitens. Fort de ce qui précède, il est établi que dans la sylviculture en plantation de Pericopsis elata indépendamment de la qualité de la station, les fortes densités sont positivement corrélées à la croissance des tiges. Autrement dit, la surface terrière et par ricochet le diamètre augmente avec la densité initiale des plantations. Ce résultat est en parfait accord avec les résultats des travaux de Groot et Cortini (2016) sur les peuplements de l'épinette noire âgé de 30 ans.
Toutefois, cette conclusion est en contradiction avec les travaux de plusieurs auteurs à l'instar de Périé et al. (2006) et Gagné et al. (2012) qui ont démontré que les faibles densités favorisent la croissance en diamètre. Cependant, les travaux de Deleuze et al. (2004) et Farooq et al. (2019) ont relevé un effet non significatif de la densité sur la croissance des tiges. Deux principales raisons peuvent expliquer les conclusions divergentes qui se dégagent des travaux cités et de la présente étude. La première explication est liée à la nature de la distribution spatiale des tiges dans les plantations. En effet et en accord avec les travaux de plusieurs auteurs, la distribution aléatoire des arbres dans les plantations peut expliquer la forte variabilité des conditions de croissance (Sokpon et Ouinsavi, 2004). La variabilité de croissance traduit en filigrane la difficulté de caractériser la relation densitécroissance des tiges. La seconde explication repose à la fois sur le poids de la densité et sur l'âge. En effet, les études susmentionnées, qui sont contraires aux conclusions du présent travail, ont été menées pour des densités initiales fortes, globalement supérieures à 1111 tiges/ha, et dont l'âge moyen était estimé à une vingtaine d'années. Cette réalité laisse penser que les phénomènes observés dans ces études peuvent changer de tendance ou de direction pour des densités bien moins inférieures à 1111 tiges/ha d'une part et pour des plantations âgées de plus de 40 ans comme c'est le cas pour les présentes plantations de Pericopsis elata. Ainsi, on pourrait énoncer sous la forme d'un axiome qu'en sylviculture de plantation de Pericopsis elata, il existe un seuil de densité initiale au-dessus duquel la croissance des tiges augmente avec la diminution de celle-ci.

Pour mieux appréhender le principe qui sous-tend les différences de croissance observées entre les plantations des grands layons et celle de recrû, il faut mettre ensemble les travaux de plusieurs auteurs et faire des postulats. Le premier postulat est basé sur le fait que la croissance est sous l'influence de trois principaux paramètres dont l'âge de 
l'arbre, les caractéristiques du site et la compétition entre les arbres (Pretzsch et al., 2002). Le deuxième postulat est basé sur le fait que des densités de plantation élevées peuvent engendrer la compétition inter et intra spécifique (Ngueguim et al., 2015). A la suite de ces postulats, précisons que les trois plantations de la RFDD sont équiennes, mono spécifiques et surtout mises en place sur la même station. Ces trois éléments mis en ensemble, on se rend compte que les seuls paramètres pouvant influencer la croissance est la compétition des tiges de Pericopsis elata entre elles d'une part, et la compétition entre ces dernières et les autres végétaux d'autre part. Les plantations ayant été abandonné après leur mise en place, la compétition est donc naturellement plus forte dans les plantations des grands layons comparée à la plantation de recrû. En d'autres termes, malgré les faibles densités présentes dans les plantations des grands layons par rapport à la plantation de recrû, la croissance des tiges est particulièrement ralentie par la forte concurrence exercée par la végétation immédiate. En effet, les plantations de grands layons aidés malheureusement par le fait qu'elles ont été abandonnées, se comportent comme des forêts naturelles. Ainsi, la croissance des tiges d'Assamela est freinée par la compétition exercée par les arbres voisins. Cette conclusion corrobore avec les travaux de Liboum et Bobo (2017) qui ont établi que la variabilité observée dans la croissance en diamètre de Baillonella toxisperma était la conséquence de la compétition interspécifique avec leurs voisins. Donc par déduction, dans le cas précis des plantations des grands layons de la RFDD (P745 et P746), la croissance a été naturellement freinée à cause des particularités propres à cette méthode sylvicole. Ainsi, il devient évident que la densité est sans effet sur la croissance dans les plantations des grands layons. Autrement dit, pour la sylviculture en plantation de Pericopsis elata, les fortes densités ne sont pas principalement une contrainte majeure pour une croissance optimale des tiges.

\section{Concurrence asymétrique et effet du milieu}

$\mathrm{La}$ forme des semi-variogrammes expérimentaux montre que les premiers points des semi-variogrammes indépendamment de la direction ne sont pas les plus élevés. En outre, on observe également une augmentation de la pente de chaque semi-variogramme. Cela traduit le fait que la concurrence n'est pas l'interaction majeure qui existe entre les tiges dans les plantations (McIntire et Fajardo, 2009). Dans le même ordre d'idées, et en tenant compte du fait que la croissance en diamètre augmente dans le même sens que la densité des plantations, on peut affirmer que la compétition intra-spécifique n'est pas une contrainte pour la croissance des tiges. Autrement dit, la compétition entre les tiges dans les plantations existe certes, mais n'a pas d'effet limitant pour la croissance de ces tiges. En d'autres termes encore, la concurrence entre les tiges existe sans toutefois infléchir sur la courbe de la croissance des tiges.

En outre, les valeurs des indices de Hegyi confortent le fait que l'effet de la concurrence est très limité dans les trois plantations. En effet, on constate bien que les indices de compétition par les dominants dans les trois plantations sont tous inférieurs à l'unité. Par ailleurs, et pour le cas spécifique de la plantation P741, la variabilité de la portée en fonction des variables explicatives traduit le fait que la compétition entre les tiges est asymétrique. En effet, on a bien constaté que la portée pour la variable mesurée $\operatorname{Dhp~}(3,9 \mathrm{~m})$ est différente de celle de la hauteur $(4,6 \mathrm{~m})$. Si on décompose la croissance en deux variantes, une horizontale et l'autre verticale, on peut ainsi conclure que la croissance en diamètre n'est pas encore concernée par les effets de la concurrence contrairement à la croissance en hauteur. Autrement dit, à ce stade de croissance pour les trois plantations, la compétition asymétrique est plus importante que la compétition symétrique. En intégrant le fait 
que l'écartement minimal entre les tiges pour la plantation P741 est de $4 \mathrm{~m}$, on peut conclure alors que la compétition symétrique est non significative. En d'autres termes et fort de ce qui précède, nous pouvons conclure que la concurrence entre les tiges de Pericopsis elata à 44 ans est principalement asymétrique. Autrement dit, à ce stade de développement des plantations de Pericopsis elata, la compétition au-dessus du sol, c'est-à-dire pour la lumière, est la plus importante que celle pour l'eau et les autres valeurs nutritives du sol. Cette conclusion est consolidée par le caractère héliophile de Pericopsis elata. Ce résultat va dans le même sens que les travaux de Montgomery et al. (2010) qui, ont abouti à la conclusion que la compétition au-dessus du sol a plus effet dans l'accumulation de la biomasse plus que la compétition sous le sol.

Par ailleurs, la forme globale des semivariogrammes expérimentaux indique également une combinaison d'autres facteurs avec la compétition. En effet, les distributions spatiales des tiges dans chaque plantation d'une part, et le caractère non significatif de la qualité de la concurrence suivant le statut social d'autre part, démontrent qu'il y a une combinaison de la concurrence avec l'effet du milieu. En effet, la forte croissance en diamètre observée pour des densités élevées se présente comme la résultante des interactions équilibrées dans les plantations. Concrètement, le mot équilibre doit être perçu ici non pas comme l'inexistence de certains effets dans les plantations mais comme la non prédominance d'un effet sur l'autre. Dans le cas de la présente étude et une fois de plus sur la base de l'allure des semi-variogrammes expérimentaux, l'effet du milieu affecte les effets de la concurrence. En effet, lorsque les arbres voisins ont des dimensions voisines au moment de la mesure, c'est qu'il y a soit phénomène de coopération, soit effet du milieu en l'absence de concurrence, soit prépondérance de l'effet du milieu sur la concurrence. Autrement dit, le niveau de croissance des tiges peut être mieux expliqué par les effets du milieu que par la concurrence entre les tiges. Ce résultat rejoint les travaux de Neufeld et al. (2014) qui avaient conclu pour l'épinette blanche, que le carbone du sol et l'humidité du sol expliquait nettement mieux la variation de la croissance que les facteurs de compétition. Dans le même ordre d'idée, Gnanguenon-Guesse et al. (2017) concluaient que la croissance des paramètres dendrométriques du teck (Tectona grandis) en plantation variait significativement en fonction du type de sol. Cela dit, dans le cadre de la présente étude et en accord avec les résultats obtenus, nous ne pouvons pas évoquer le phénomène de coopération en termes de croissance en diamètre au regard de la valeur de la portée des semi-variogrammes théoriques. Autrement dit, il s'agit principalement de la prédominance de la qualité de la station sur la concurrence.

\section{Conclusion}

Les présents travaux avaient pour objectif de comprendre les conditions de croissance de Pericopsis elata dans les plantations de la RFDD au passé sans traitements sylvicoles et induire les propositions d'aménagement susceptibles d'améliorer leur suivi. Les formes des semivariogrammes expérimentaux obtenues permettent d'affirmer qu'en l'absence des traitements sylvicoles appropriés, les effets du milieu ont plus d'impact sur la croissance que la concurrence intra spécifique. Ainsi, nous pouvons conclure que concernant les interactions entre les tiges de Pericopsis elata dans les plantations de Deng-Deng, on assiste à la primauté de l'effet du milieu sur la concurrence. Autrement dit, notre hypothèse de départ n'est pas confirmée, car les densités n'ont pas principalement d'effet limitant sur la croissance contrairement aux effets du milieu qui se sont révélés comme étant le facteur qui a plus d'impact sur la croissance des tiges. En outre, au regard des faibles valeurs de la compétition exercée par les dominants, il devient évident que la compétition entre les tiges voisines est relativement faible. 
Toutefois, le mode de compétition qui influence particulièrement la croissance des tiges des plantations de Pericopsis elata âgées de 44 ans est la compétition asymétrique. Les distributions spatiales des tiges dans chaque plantation, ajoutées aux performances de la croissance en rapport avec les densités, permettent de conclure que dans la sylviculture en plantation de Pericopsis elata, les fortes densités induisent une meilleure croissance des tiges. Par ailleurs, on peut affirmer que la méthode sylvicole la plus appropriée pour Pericopsis elata est la méthode de recrû. Compte tenu de ce qui précède, nous suggérons que des éclaircies de rattrapage, orientées vers un réel équilibre entre la densité et l'espacement entre les arbres, soient effectuées dans les plantations de Pericopsis elata de la RFDD afin de réduire considérablement la concurrence intra spécifique dans la plantation P741 et les concurrences intra et interspécifiques dans les plantations de grands layons.

\section{CONFLIT D'INTERETS}

Les auteurs déclarent qu'ils n'ont aucun conflit d'intérêts concernant ce travail.

\section{CONTRIBUTIONS DES AUTEURS}

JA a conçu le protocole de collecte des données et rédigé le manuscrit. SBK et Antoine AMZ ont supervisé la rédaction et procédé à la relecture du manuscrit. JDD a également contribué à la collecte des données.

\section{REMERCIEMENTS}

Les auteurs remercient les vaillantes populations de Yebi et leur chef pour leur accueil. Dans le même ordre d'idée nous adressons notre sentiment de gratitude à l'antenne de l'Institut de Recherche Agricole pour le Développement (IRAD) de Belabo. Ce travail a bénéficié du soutien de l'Agence National d'Appui au Développement Forestier (ANAFOR) à travers le programme OIBT/CITES sur la gestion durable de l'Assamela.

\section{REFERENCES}

Akouehou GS, Lokossou RS, Lègba IS, Bio IC, Yaou LZ. 2017. Dynamique des peuplements des Parcelles d'Essais Sylvicoles (PES) de la forêt classée de la Sota au Nord-Est du Bénin. International Journal of Biological and Chemical Sciences, 11(5): 1994-2004. DOI: $10.4314 /$ ijbcs.v11i5.5.

Bérubé-Deschênes A, Franceschini T, Schneider R. 2017. Quantifying competition in white spruce (Picea glauca) plantations. Annals of Forest Science, 74: 26. DOI: 10.1007/s13595017-0624-3.

Bourland N, Kouadio YL, Fétéké F, Lejeune P, Doucet JL. 2012. Ecology and management of Pericopsis elata (Harms) Meeuwen (Fabaceae) populations. Biotechnologie, Agronomie, Société et Environnement, 16(4): 486-498. DOI: http://hdl.handle.net/2268/135821

Bourland N. 2013. Dynamique d'une espèce ligneuse héliophile longévive dans un monde changeant : le cas de Pericopsis elata (Harms) Meeuwen (Fabaceae) au Sud-Est du Cameroun. Thèse de Doctorat, Université de Liège, Belgique, $140 \mathrm{p}$.

Deleuze C, Pain O, Dhôte JF, Hervé JC. A flexible radial increment model for individual trees in pure even-aged stands. Annals of Forest Science, 61(4): 327-335. DOI: $10.1051 /$ forest:2004026

FAO. 2011. State of the World's Forest 2011. Food and Agriculture Organization of the United Nations (FAO), Roma. http://www.fao.org/3/i2000e/i2000e00.ht $\mathrm{m}$.

Farooq TH, Wu W, Tigabu M, Ma X, Lui Z, Rashid MHU, Gilani MM, Wu P. 2019. Growth, Biomass Production and Root Development of Chinese fir in Relation to Initial Planting Density. Forests, 10(3): $\quad 236 . \quad$ DOI: 10.3390/f10030236 
Gagné L, Lavoie L, Binot JM. 2012. Growth and mechanical properties of wood after commercial thinning in a 32-year-old white spruce (Picea glauca) plantation. Canadian Journal of Forest Research, 42(2): $\quad$ 291-302. DOI: 10.1139/x11-181

Gnanguenon-Guesse D, Nounagnon GS, Aoudji AKN, Ganglo JC. 2017. Étude des caractéristiques structurales des teckeraies en fonction de l'âge et du type de sol au Sud et au Centre Bénin. International Journal of Biological and Chemical Sciences, 11(5): 2119-2132. DOI: 10.4314/ijbcs.v11i5.15

Grison F. 1979. Les plantations d'Assamela (Pericopsis elata) en forêt semi-décidue Camerounaise. CIRAD: GERDATCTFT. https://agritrop.cirad.fr/361146/.

Groot A, Cortini F. 2016. Effects of initial planting density on tree and stand development of planted black spruce up to age 30. The Forestry Chronicle, 92(2): 200-210. DOI: 10.5558/tfc2016-039

Hegyi F. 1974. A simulation model for managing jack-pine stands. In: Growth model for tree and stands simulation. Royal College of Forestry, Stockholm. Research Note, 30: 74-90.

Kouassi MA, Okaingni JC, Baka D, Lasm T, Kouame FK, Biemi J. 2012. Application des méthodes statistiques et géostatistiques à l'étude de la conductivité électrique des eaux souterraines de la région du N'Zi-Comoé (Centre-Est de la Côte d'Ivoire). International Journal of Biological and Chemical Sciences, $6(2)$ : 897-912. DOI: 10.4314/ijbcs.v6i2.31

Kuyper TW, Onguene NA, Van Gemerden BS, Mbelli H. 2001. Integration of ecological knowledge in sustainable management of rainforests of south Cameroon, with special reference to myorrhyzia associations. In Sustainable Management of African Rain Forest (ed). The
Tropenbos Foundation: Wageningen; 5058.

Le Moguédec G, Dhôte JF. 2012. Fagacées: a tree-centered growth and yield model for sessile oak (Quercus petraea L.) and common beech (Fagus sylvatica L.). Annals of Forest Science, 69(2): 257269.

DOI: https://doi.org/10.1007/s13595-0110157-0

Liboum M, Bobo KS. 2017. Analyse de l'effet de la concurrence végétale sur la croissance du Moabi en forêt dense tropicale perturbée de l'EstCameroun. International Journal of Biological and Chemical Sciences, 11(6): 2671-2692. DOI: 10.4314/ijbcs.v11i6.10

McIntire EJ, Fajardo A. 2009. Beyond description: the active and effective way to infer processes from spatial patterns. Ecology, 90(1): 46-56. DOI: https://doi.org/10.1890/07-2096.1

Medhurst JL, Beadle CL, Neilsen WA. 2001. Early-age and later-age thinning affects growth, dominance, and intraspecific competition in Eucalyptus nitens plantations. Canadian Journal of Forest Research, 31(2): $\quad$ 187-197. DOI: https://doi.org/10.1139/x00-163

Montgomery RA, Reich PB, Palik BJ. 2010. Untangling positive and negative biotic interactions: views from above and below ground in a forest ecosystem. Ecology, 91(12): $3641-3655$. DOI: https://doi.org/10.1890/09-1663.1

Neufeld BA, Morris DM, Luckai N, Reid DEB, Bell FW, Shahi C, Meyer WL, Adhikary S. 2014. The influence of competition and species mixture on plantation-grown white spruce: Growth and foliar nutrient response after 20 years. The Forestry Chronicle, 90(1): 70-79. DOI: 10.5558/tfc2014-012

Ngueguim JR, Betti JL, Riera B, Ambara J, Tchatat M, Onana J. 2012. Growth and productivity of Pericopsis elata (Harms) Meeuwen in some forest plantations of 
Cameroon. Forest Science and

Technology, 8(1): 1-10. DOI: 10.1080/21580103.2012.658234

Ngueguim JR, Zapfack L, Noiha NV, Onana D, Betti JL, Riera B. 2015. Expériences sylvicoles au Cameroun : Croissance, mortalité et adaptabilité des espèces de bois d'œuvre dans la station forestière de Mangombé (1964-2010). International Journal of Biological and Chemical Sciences, 9(6): 2789-2807. DOI: 10.4314/ijbcs.v9i6.22.

Périé C, Ouimet R, Duchesne L. 2006. Evolution Contemporaine des principales caractéristiques dendrométriques des stations du Réseau d'Etude et de Surveillance des Ecosystèmes Forestiers (RESEF). Ministère des Ressources naturelles et de la Faune, Direction de la recherche forestière, Québec (Québec) Canada.

Pretzsch H, Schütze G. 2016. Effect of tree species mixing on the size structure, density, and yield of forest stands. European Journal of Forest Research, 135(1): 1-22. DOI: https://doi.org/10.1007/s10342-0150913-z

Pretzsch H, Biber P, Duurský J. 2002. The single tree-based stand simulator SILVA: construction, application and evaluation. Forest Ecology and Management, 162(1): 3-21. DOI: 10.1016/S0378-1127(02)00047-6

Pretzsch H, Biber P. 2010. Size-symmetric versus size-asymmetric competition and growth partitioning among trees in forest stands along an ecological gradient in central Europe. Canadian Journal of Forest Research, 40(2): 370-384. DOI: 10.1139/X09-195

R Core Team. 2020. R: A language and environment for statistical computing. $\mathrm{R}$ Foundation for Statistical Computing, Vienna, Austria. URL https://www.Rproject.org/.

Sokpon N, Ouinsavi C. 2004. Gestion des plantations de Khaya senegalensis au Bénin. Bois \& Forêts des Tropiques, 279(1): $\quad 37-46 . \quad$ DOI: https://doi.org/10.19182/bft2004.279.a20 247.

Tchingsabe O, Mbile JN, Dibong SD, Tchatat M, Ngomeni AF. 2017. Évaluation du potentiel des bois d'œuvre des parcelles Permanentes d'observation du bloc Kébé, Bélabo (Est-Cameroun). Journal of Applied Biosciences, 116: 11601-11611. 